\title{
Implementación del OEE en el proceso de fabricación de vidrio templado
}

\section{OEE implementation in the tempered glass manufacturing process}

\author{
GONZÁLEZ-SÓBAL, Martín†*, CALDERÓN-PALOMARES, Luis Antonio y GÓMEZ-MÁRQUEZ, \\ Montserrat
}

Tecnológico Nacional de México / Instituto Tecnológico Superior de Huatusco, Av. 25 poniente \#100, Colonia Reserva Territorial, CP. 94100

ID $1^{\text {er }}$ Autor: Martín, González-Sóbal / ORC ID: 0000-0003-0038-8319, Researcher ID Thomson: N-6243-2018, CVU CONACYT ID: 463431

ID $1^{\text {er }}$ Coautor: Luis Antonio, Calderón-Palomares / ORC ID: 0000-0001-9846-5567, Researcher ID Thomson: N-62592018, CVU CONACYT ID: 238274

ID $2^{\text {do }}$ Coautor: Montserrat, Gómez-Márquez, / ORC ID: 0000-0002-2901-1745, Researcher ID Thomson: S-8535-2018, CVU CONACYT: 999026

DOI: $10.35429 /$ JOTI.2019.10.3.11.21

Recibido 27 de Abril, 2019; Aceptado 15 de Junio, 2019

\section{Resumen}

Objetivo: Implementar el OEE como indicador que permita evaluar y mejorar el proceso de fabricación de vidrio templado. Metodología: Se analizan las líneas de producción para determinar los índices: disponibilidad, ritmo y calidad. Se obtiene una primera evaluación, con un rendimiento global de $35 \%$ para el mes de octubre de 2018, dicho resultado coloca a las líneas de producción en un rango de "inaceptable" en la escala global de dicho índice, lo cual implica que la organización tiene un alto potencial de mejora. Es en este punto donde se determinan estrategias que permitirán mejorar dicho rendimiento. En primer lugar se analizan los flujos de proceso de ambas líneas, identificando áreas de mejora y simplificando etapas de fabricación, lo cual permite optimizar tiempos de producción y disponibilidad de los equipos, una segunda acción fue la modificación del equipo de corte, permitiendo procesar tres tipos de parte, sin necesidad de hacer cambios en la herramienta, lo cual disminuye tiempos de preparación. Finalmente se evalúo y mejoró las condiciones de iluminación, con esto reducimos los tiempos de inspección en la calidad del producto. Contribución: Las acciones implementadas permitieron obtener un incremento del 5\% del OEE, en relación a la medición anterior.

OEE, rendimiento, proceso

\begin{abstract}
Resumen
Objective: Implement the OEE as an indicator that allows evaluating and improving the manufacturing process of tempered glass. Methodology: Production lines are analyzed to determine the indexes: availability, pace and quality. A first evaluation is obtained, with an overall yield of $35 \%$ for the month of October 2018, this result places the production lines in a range of "unacceptable" on the global scale of said index, which implies that the organization It has a high potential for improvement. It is at this point that strategies are determined that will improve said performance. Firstly, the process flows of both lines are analyzed, identifying areas for improvement and simplifying manufacturing stages, which allows to optimize production times and equipment availability, a second action was the modification of the cutting equipment, allowing three processes to be processed Part types, without the need to make changes to the tool, which reduces preparation times. Finally, the lighting conditions were evaluated and improved, with this we reduce the inspection times in the quality of the product. Contribution: The actions implemented allowed obtaining an increase of 5\% of the OEE, in relation to the previous measurement.
\end{abstract}

OEE, performance, process

Citación: GONZÁLEZ-SÓBAL, Martín, CALDERÓN-PALOMARES, Luis Antonio y GÓMEZ-MÁRQUEZ, Montserrat. Implementación del OEE en el proceso de fabricación de vidrio templado. Revista de Invención Técnica 2019. 3-10:11-21

\footnotetext{
*Correspondencia al Autor (Correo Electrónico: martinglezs@itshuatusco.edu.mx)

$\dagger$ Investigador contribuyendo como primer autor.
} 


\section{Introducción}

Este proyecto tiene como finalidad la implementación de la razón OEE (Overall Equipment Effectiveness o Eficiencia General de los Equipos), dentro de las líneas de producción de vidrio templado, con el fin de que sirva de guía en la mejora de los procesos y contar con una métrica respecto al uso de los recursos empleados en la producción.

La investigación se desarrolla en una industria ubicada en el Estado de México, por razones de confidencialidad se omite el nombre de ella, así como los procesos analizados se identifican con abreviaturas.

Actualmente dicha organización presenta varios problemas en sus procesos productivos como: tiempos de paro por mantenimiento, alto porcentaje en desperdicio de material, producto defectuoso y bajo rendimiento en las líneas de producción. Para tratar de atacar los problemas anteriores se propone la implementación del OEE, ya que al ser un indicador de la efectividad global del equipo, integra tres indicadores importantes que son: el ritmo de producción, la disponibilidad y la calidad.

En primer lugar se realiza un análisis FODA que permita identificar el escenario interno y externo que guarda la empresa actualmente, posteriormente se realiza un análisis del proceso de producción de vidrio templado, identificando las etapas y los equipos involucrados en el proceso, con el fin de identificar aquellas actividades que no aportan valor y que en consecuencia generan bajo un desempeño en los indicadores que engloban al OEE.

A continuación se diseñan plantillas en hojas de cálculo para hacer la recolección de datos que permitan determinar los índices de desempeño, poder tener una primera estimación del OEE y hacer una evaluación sobre las áreas de oportunidad en las que se puede incursionar. Derivado de esto, se proponen dos estrategias principales, la primera que consiste en la reducción de tiempos simplificando la ejecución de actividades, y como segunda la mejora tecnológica en el equipo de corte, adecuando el herramental para que pueda procesar dos tipos de parte, reduciendo los tiempo de cambio.
Una vez implementadas dichas acciones, se procede a realizar una nueva medición de los indicadores y se determina el desempeño global del OEE, encontrando una mejora del $5 \%$ en relación al mes anterior.

\section{Revisión de literatura}

La medición del desempeño de las áreas internas que conforman un proceso productivo permite conocer en tiempo real el estado, la evolución y las problemáticas asociadas, de manera que es posible pronosticar fallas con el fin de generar acciones oportunamente. Medir permite planificar con mayor certeza y confiabilidad los eventos, procesos y procedimientos en toda área interna productiva de la industria. La medición del desempeño permite identificar con mayor precisión las oportunidades de mejora de un proceso dado, incluyendo el análisis y justificación del origen de los eventos. Una de las razones más importantes radica en la necesidad de conocer a fondo los procesos administrativos, técnicos, de producción y apoyo que se den en la industria para gestionar su mejoramiento.

Un indicador es una magnitud que expresa el comportamiento o desempeño de un proceso referenciado en variables cuantitativas o cualitativas, que al compararse con algún nivel de referencia permite detectar desviaciones positivas o negativas. También es la conexión de dos medidas relacionadas entre sí, que muestra la relación en ellas (Mejía Nieto, 2012). Trabajar con indicadores exige disponer de todo un sistema que abarque desde la adquisición de los datos que caractericen la ocurrencia del evento hasta la retroalimentación de las decisiones que permiten mejorar los procesos. Los indicadores pueden ser valores, unidades, índices, etc.; es decir, factores que permiten establecer el logro y el cumplimiento de la misión, objetivos estratégicos y metas de una organización.

Antes de realizar la implementación de los indicadores en un estudio de seguimiento y medición de desempeño en una compañía, deben tenerse en cuenta las siguientes consideraciones:

- Lo que no se puede medir, no se puede controlar.

- Lo que no se puede medir, no existe. 
- Los indicadores son un medio y no un fin.

- Actualidad: es un valor fluctuante basado en la medición acerca de lo que se hace en el presente con los recursos y restricciones existentes.

- Capacidad: es un valor fijo, significa lo máximo que podría hacerse con los recursos existentes y bajo las restricciones presentes. Es importante hacer explícitos los recursos y restricciones que se relacionan con la capacidad máxima.

- Potencialidad: es lo máximo que se puede obtener si se desarrollan los recursos y se remueven los "cuellos de botella" para mejorar la capacidad. (Mora, 2011).

\section{OEE (Overall Equipment Effectiveness)}

El OEE (Overall Equipment Effectiveness o Eficiencia General de los Equipos), es una razón que mide la eficiencia de los equipos industriales, tiene una gran ventaja ya que también se puede interpretar como medición global de tres índices importantes que son: la disponibilidad, el ritmo de producción y la calidad.

De acuerdo a (Cruelles, 2010) el tener un OEE de, por ejemplo el $40 \%$ significa que de cada 100 piezas buenas que la maquina podría haber producido, sólo ha producido 40.

Se dice que engloba todos los parámetros fundamentales, porque del análisis de los tres índices que integran al OEE, es posible saber si lo que falta hasta el $100 \%$ se ha perdido por disponibilidad (la maquinaria estuvo cierto tiempo parada), eficiencia o ritmo de producción (la maquinaria estuvo funcionando a menos de su capacidad total) y calidad (se han producido unidades defectuosas). (Cruelles, 2010)

Los indicadores anteriores los calculamos de la siguiente manera:

Disponibilidad $=$

Tiempo de operación disponible

Tiempo de operación total
Ritmo $=\frac{\text { Producción total }}{\text { Producción potencial }}$

Calidad $=\frac{\text { Producción de calidad producida }}{\text { Producción total }}$

$O E E=$ Disponibilidad $*$ Ritmo $*$ Calidad $(4)$

$\mathrm{Si}$ integramos a estos factores como subconceptos de un concepto principal vamos a encontrar la fundamentación de esta ecuación y con ello tendremos el marco de seguridad que nos permite tomar el conocimiento como seguro.

La efectividad global del equipamiento de una planta es un problema complejo y por lo tanto sus elementos no tienen relaciones causaefecto unívocas y por ello funcionan en una conjunción donde si uno de los elementos se hiciera cero todos los demás elementos no podrían compensar su falta. (Belohlavek, 2006).

El OEE se ha determinado en diversos tipos de industrias a nivel mundial, con el fin de establecer un punto de comparación basado en una herramienta que trasciende más allá de la particularidad de cada sector en su proceso productivo. En la siguiente tabla se muestran las diferentes categorías de acuerdo al desempeño obtenido del OEE en la industria:

\begin{tabular}{|c|c|c|c|c|}
\hline \multicolumn{2}{|l|}{ \% OEE } & Calificación & $\begin{array}{l}\text { Avance hacia } \\
\text { clase mundial }\end{array}$ & Competitividad \\
\hline $\begin{array}{l}\text { OEE } \\
65 \%\end{array}$ & $<$ & Inaceptable & $\begin{array}{l}\text { Se producen } \\
\text { importantes } \\
\text { pérdidas } \\
\text { económicas. }\end{array}$ & $\begin{array}{l}\text { Muy baja } \\
\text { competitividad. }\end{array}$ \\
\hline $\begin{array}{l}65 \% \\
\text { OEE } \\
75 \%\end{array}$ & $\begin{array}{l}< \\
<\end{array}$ & Regular & $\begin{array}{ll}\text { Aceptable sólo } \\
\text { si se está en } \\
\text { proceso } & \text { de } \\
\text { mejora. } & \\
\text { Pérdidas } & \\
\text { económicas. } & \end{array}$ & $\begin{array}{l}\text { Baja } \\
\text { competitividad. }\end{array}$ \\
\hline $\begin{array}{l}75 \% \\
\text { OEE } \\
85 \%\end{array}$ & $\begin{array}{l}< \\
<\end{array}$ & Aceptable & $\begin{array}{lr}\text { Continuar } & \text { la } \\
\text { mejora } & \text { para } \\
\text { superar el } & 85 \% \\
\text { y avanzar hacia } \\
\text { la } & \text { clase } \\
\text { mundial. } & \\
\text { Ligeras } & \\
\text { pérdidas } & \\
\text { económicas. }\end{array}$ & $\begin{array}{l}\text { Competitividad } \\
\text { ligeramente } \\
\text { baja. }\end{array}$ \\
\hline $\begin{array}{l}85 \% \\
\text { OEE } \\
95 \%\end{array}$ & $\begin{array}{l}< \\
<\end{array}$ & Buena & $\begin{array}{l}\text { Entra en valores } \\
\text { de } \quad \text { clase } \\
\text { mundial. }\end{array}$ & $\begin{array}{l}\text { Buena } \\
\text { competitividad. }\end{array}$ \\
\hline $\begin{array}{l}\text { OEE } \\
95 \%\end{array}$ & $>$ & Excelencia & $\begin{array}{l}\text { Valores de clase } \\
\text { mundial. }\end{array}$ & $\begin{array}{l}\text { Excelente } \\
\text { competitividad. }\end{array}$ \\
\hline
\end{tabular}

Tabla 1 Escala de evaluación del OEE. Fuente: Una herramienta de Mejora, El OEE, Alonso G. Hugo L., Universidad de Holguín Oscar Lucero Moya, 2009. 


\begin{abstract}
Análisis FODA
El análisis FODA tiene sus orígenes en la obra Business Policy, Text and Cases (1965), escrita por cuatro profesores de la Universidad de Harvard, Edmund Philip Learned (1900-1991), Roland Chris Christensen (1919-1999), Kenneth Richmond Andrews (1916-2005) y William D. Guth. Este análisis es uno de los primeros modelos que se interesan por el entorno externo una organización. Hasta entonces, los modelos de estrategia tenían tendencia a limitarse a la planificación estratégica, sin tener en cuenta realmente su entorno.
\end{abstract}

Hoy en día, el análisis FODA se usa principalmente en los departamentos de marketing de las grandes empresas, pero también hay muchas PYMES que lo emplean como herramienta de ayuda en la toma de decisiones. (Speth, 2016)

Las variables que contempla el análisis FODA comprende los siguientes conceptos: fortalezas, oportunidades, debilidades y amenazas. A continuación se describe en que consiste cada uno de ellos:

- Fortalezas: es algo en lo que la organización es competente, se traduce en aquellos elementos o factores que estando bajo su control, mantiene un alto nivel de desempeño, generando beneficios. Las fortalezas pueden asumir diversas formas como: recursos humanos maduros, capaces y experimentados, habilidades y destrezas importantes para hacer algo, activos físicos valiosos, finanzas sanas, etc.

- Debilidades: significa una deficiencia o carencia, algo en lo que la organización tiene bajos niveles de desempeño y por tanto es vulnerable, denota una desventaja ante la competencia, con posibilidades pesimistas para el futuro. $\mathrm{Al}$ igual que las fortalezas éstas pueden manifestarse a través de sus recursos, habilidades, tecnología, organización, productos, etc.
- Oportunidades: son aquellas circunstancias del entorno que son potencialmente favorables para la organización y pueden ser cambios o tendencias que se detectan y que pueden ser utilizados ventajosamente para alcanzar o superar los objetivos. Las oportunidades que pueden presentarse en cualquier ámbito, como el político, económico, social, tecnológico, etc., dependiendo de la naturaleza de la organización, pero en general, se relacionan principalmente con el aspecto mercado de una empresa.

- Amenazas: son factores del entorno que resultan en circunstancias adversas que ponen en riesgo el alcanzar los objetivos establecidos, pueden ser cambios o tendencias que se presentan repentinamente o de manera paulatina, las cuales crean una condición de incertidumbre e inestabilidad en donde la empresa tiene muy poca o nula influencia, las amenazas también, pueden aparecer en cualquier sector como en la tecnología, competencia, nuevos productos, políticas gubernamentales, impuestos, etc. (Ramírez, 2009)

\section{Metodología}

Para el desarrollo de este proyecto se implementaron una serie de etapas, las cuales se describen a continuación:

\section{Análisis FODA.}

Antes de iniciar con el desarrollo del proyecto se decide implementar este análisis ya que permite tener un diagnóstico preciso del funcionamiento interno y externo de la organización, para su realización se contó con la participación de las áreas directivas y los jefes de los principales departamentos relacionados con el área de producción. Dicho análisis se presenta a continuación: 


\begin{tabular}{|c|c|}
\hline Fortalezas & Debilidades \\
\hline $\begin{array}{l}\text { La organización es dinámica, } \\
\text { busca el crecimiento y } \\
\text { desarrollo. } \\
\text { Está abierta al cambio y las } \\
\text { mejoras. } \\
\text { Se cuenta con productos de } \\
\text { calidad. }\end{array}$ & $\begin{array}{l}\text { No se encuentran definidas } \\
\text { claramente las } \\
\text { responsabilidades. } \\
\text { Alto porcentaje de } \\
\text { desperdicio. } \\
\text { Mala comunicación en las } \\
\text { áreas operativas. }\end{array}$ \\
\hline Amenazas & Oportunidades \\
\hline $\begin{array}{l}\text { Empresas competidoras } \\
\text { incursionen en el mismo } \\
\text { mercado. } \\
\text { Perder nuevos segmentos de } \\
\text { mercado. }\end{array}$ & $\begin{array}{l}\text { Incursionar en otros estados } \\
\text { de la república. } \\
\text { Precios competitivos. }\end{array}$ \\
\hline
\end{tabular}

Tabla 2 Análisis FODA.

Fuente: Elaboración propia.

Del análisis anterior podemos concluir que la empresa tiene amplias oportunidades de crecer en nuevos mercados, ya que puede competir en base a un producto de calidad, aunado aún precio competitivo, sin embargo, si la empresa no optimiza sus procesos operativos y administrativos, corre el riesgo de ser desplazada por la competencia en nuevos mercados.

\section{Descripción del proceso.}

En segundo lugar se procedió a realizar un análisis del proceso de producción de vidrio templado, el cual consta de las siguientes etapas:

Corte: L-2 es la maquina utilizada para el corte de vidrio monolítico, la función de esta máquina es realizar trazos perfectos sobre la lámina de vidrio, el carro de corte y da precisión al mismo mediante una rulina de diamante.

Desfilado: G-A es la máquina que realiza desbaste a los filos del vidrio, para darle presencia al mismo. Consiste en desbastar los lados del vidrio mediante dos muelas de diamante.

Canteado: G-B es la máquina que realiza detalle curvo a los filos del vidrio.

Lavado: cuando hablamos de desbaste del vidrio, se considera que se expulse una merma que en el ámbito de corte de vidrio se conoce como polvo de vidrio, que debe ser removido del mismo, por lo que la máquina Billco - Danger realiza este trabajo de lavado para que no se dañe el vidrio con el polvo que ocasiona rayas o talladuras.
Decorado: Decoradoras Studio son utilizadas para este trabajo, en estas líneas continuas se utilizan decoradoras automáticas, su función principal es, mediante un carro de impresión y un esténcil generar el decorado por el lado aire del vidrio, y estos decorados pueden ser de varios tipos y modelos.

Horneado: la maquina Temglass realiza la parte semifinal de este proceso, su función es cambiar las propiedades del vidrio haciéndolo más resistente al choque térmico, resistente a los cambios mecánicos a tracción, flexión o torsión y le brinda mayor resistencia mecánica al impacto.

Inspección: el equipo de calidad realiza labores de inspección antes de empacar el producto final y destinarlo al cliente, se realizan inspecciones visuales y se identifican los defectos tanto en la calidad del decorado, como en la calidad del templado.

Empaque: una vez certificada por el área de calidad el producto final es empacado con papel encerado, evita que los vidrios tengan contacto directo entre si y puedan dañarse. Se empacan en cajas de madera resistente, dentro de ella se coloca una bolsa plástica y dentro de ella se coloca el vidrio de esta forma evitan que el producto final obtenga daños durante su transporte a destino final (cliente).

Almacenaje: las cajas son transportadas con el montacargas y almacenadas para su posterior entrega al cliente.

Una vez descritas las actividades que se desarrollan en la fabricación de vidrio templado, se procede a realizar el análisis de tiempos del proceso, a partir del siguiente diagrama de flujo de proceso: 


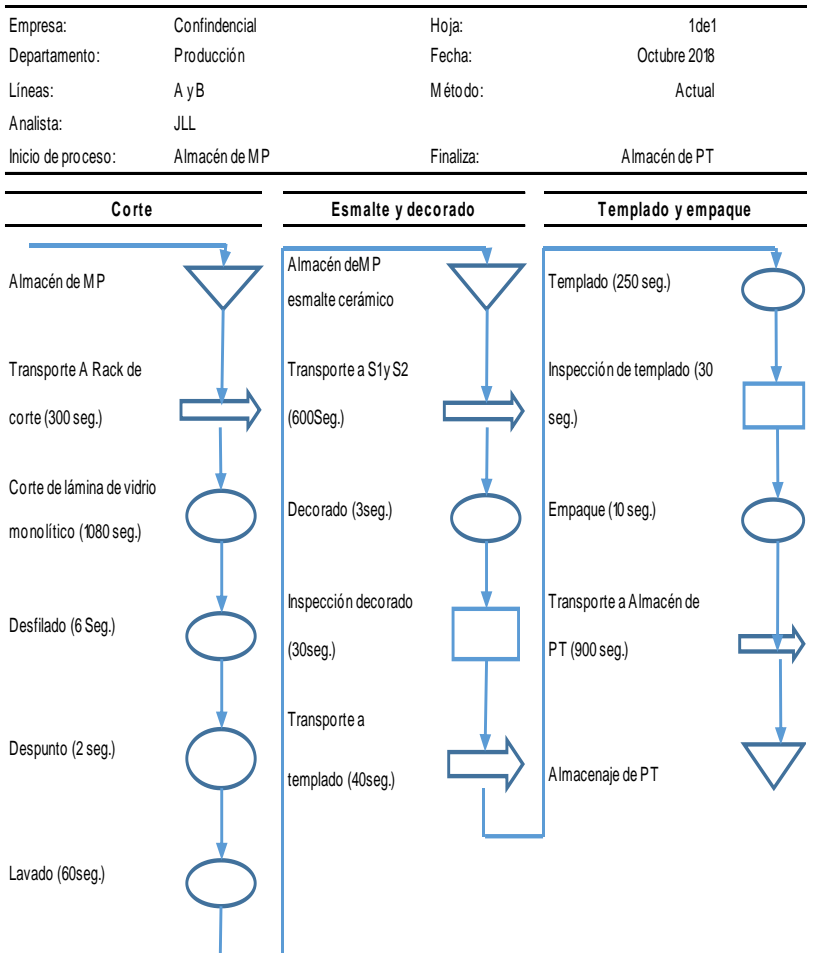

Figura 1 Diagrama de proceso

Fuente: Elaboración propia.

Del diagrama anterior podemos observar que el proceso de producción de vidrio templado se realiza en un total de 3,311 segundos, los cuales 1,840 segundos corresponde a actividades de transporte y 60 segundos a inspección, siendo las que cubren el mayor porcentaje del tiempo total, además de ser actividades que no aportan valor al proceso. Lo antes descrito lo podemos observar en el siguiente cuadro resumen:

\begin{tabular}{|c|c|c|c|}
\hline Símbolo & Descripción & Cantidad & $\begin{array}{l}\text { Tiempo } \\
\text { (seg.) }\end{array}$ \\
\hline & Operación & 7 & 1,411 \\
\hline & Transporte & 3 & 1,840 \\
\hline & Inspección & 2 & 60 \\
\hline & Demora & 0 & 0 \\
\hline & Almacenaje & 3 & 0 \\
\hline & Total & 15 & 3,311 \\
\hline
\end{tabular}

\section{Implementación del OEE.}

En tercer lugar se procede a la obtención de la información necesaria para la determinación del OEE, esta se obtendrá del reporte de producción que se genera día a día, en el cual se reportan los avances de producción por pieza y en metros cuadrados, dicho reporte se presenta a continuación:

\begin{tabular}{|c|c|c|c|c|c|c|c|}
\hline & CoI & rte $\mathrm{M}$ ar & ual & & & & \\
\hline & FPR-0 & $1-006$ & & 147,322 & |\# \# \# \# & 0 & 0 \\
\hline & Fecha / & Turno & & & Tiempc & & \\
\hline Dí: & Tur & Inic ${ }^{-}$ & $F i^{-}$ & $U t i \mp$ & M & $\nabla$ & $\nabla$ \\
\hline $2 \mathrm{E}+05$ & 1 & 10:00 & $14: 00$ & 240 & & & \\
\hline $2 \mathrm{E}+05$ & 2 & $14: 00$ & $21: 40$ & 460 & 60 & & \\
\hline $2 \mathrm{E}+05$ & 2 & 13:00 & 17:30 & 270 & 30 & & \\
\hline $2 \mathrm{E}+05$ & 3 & $21: 40$ & $06: 00$ & 480 & 30 & & \\
\hline $2 \mathrm{E}+05$ & 3 & 22:50 & 06:00 & 430 & 30 & & \\
\hline
\end{tabular}

Figura 2 Reporte de producción del área de corte. Fuente: Elaboración propia

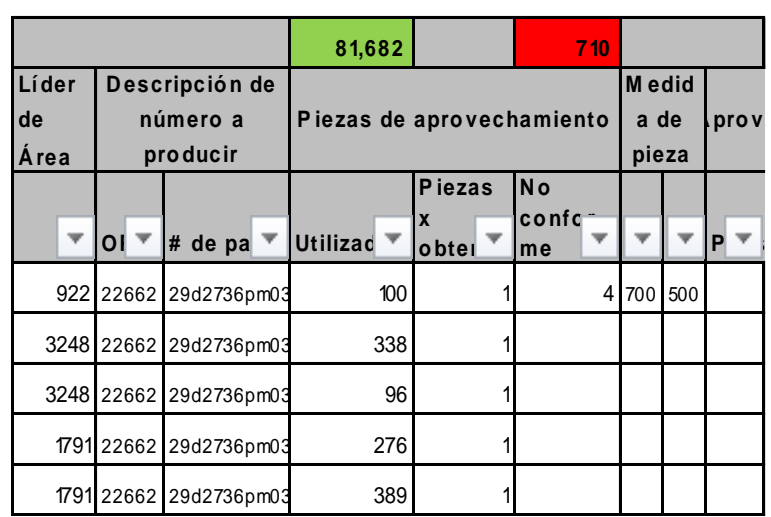

Figura 3 Reporte de aprovechamiento del área de corte Fuente: Elaboración propia

De acuerdo al formato presentado en las figuras 2 y 3 , se reporta la cantidad de piezas procesadas por inicio y termino orden de producción (OP). En las columnas se reporta la fecha en la que se está trabajando, el turno $\left(1^{\circ}, 2^{\circ}, 3^{\circ}\right)$, el inicio y fin de la OP, se cuantifican los minutos que se utilizaran en la operación para cada turno, se reporta el tiempo muerto en minutos; por mantenimiento, y por eventos de fuerza mayor, adicionalmente se presenta: código del líder de área, OP y numero de parte a procesar, piezas realizadas, piezas por obtener, y la cantidad de pieza no conformes, finalmente se reportan las dimensiones del vidrio a procesar.

Tabla 3 Cuadro resumen.

Fuente: Elaboración propia 
Con los datos que se reportan en la bitácora, se facilita la vinculación de una plantilla para el cálculo de OEE., para ello se creó una hoja de cálculo para determinar los indicadores: disponibilidad, rendimiento, calidad. Dicha hoja fue segmentada de acuerdo a las etapas del proceso, y para cada etapa se cuenta con una bitácora por separado, en el primer libro se encuentran los indicadores del área de corte, en el segundo libro se encuentra el cálculo de los indicadores de la línea $\mathrm{A}$, en el tercer libro se encuentra el cálculo de los indicadores de la línea $\mathrm{B}$, en el cuarto libro se encuentra el cálculo de los indicadores de la decoradora Studio1, en el quinto libro se encuentra el cálculo de la decoradora Studio 2, en el quinto libro se encuentra el cálculo de los indicadores del área de templado y por último en el sexto libro se muestra el resumen y promedio de estos indicadores por semana.

A continuación se presentan las hojas de cálculo creadas, mostrando los datos del primer día en el que se inicia esta plantilla, sobre el cálculo de los indicadores de corte, cabe resaltar que se aplica para el cálculo de los indicadores de las demás etapas y el resumen semanal del cálculo OEE.

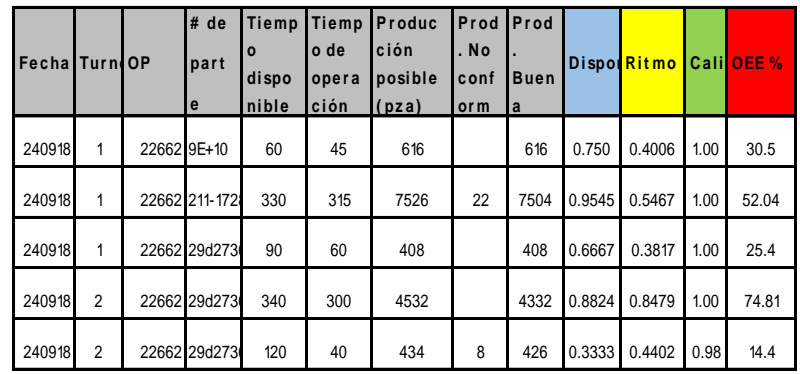

Figura 4. Formato de cálculo para indicadores OEE Fuente: Elaboración propia.

Se vinculó la bitácora mostrada en al figura 4 , en la que ya se registraban los datos anteriormente con esta nueva plantilla, los resultados de las columnas de disponibilidad, ritmo, calidad y OEE, se calculan automáticamente.

Al hacer uso de la plantilla para el cálculo de los indicadores OEE, se obtuvieron los resultados esperados para la semanas 40 a 43 que comprenden de las fechas del 01 de octubre del 2018 al 02 de noviembre del 2018. Los resultados obtenidos son los siguientes:

\begin{tabular}{|c|c|c|c|c|c|c|c|}
\hline Semana & L-2 & G-A & G-B & $\mathbf{S}-\mathbf{A}$ & S-B & Tamg. & Disp. \\
\hline $\begin{array}{l}01 / 10 / 18 \\
- \\
07 / 10 / 18\end{array}$ & $68 \%$ & $44 \%$ & $35 \%$ & $42 \%$ & $42 \%$ & $98 \%$ & $55 \%$ \\
\hline $\begin{array}{l}08 / 10 / 18 \\
- \\
14 / 10 / 18\end{array}$ & $62 \%$ & $42 \%$ & $40 \%$ & $47 \%$ & $47 \%$ & $81 \%$ & $53 \%$ \\
\hline $\begin{array}{l}15 / 10 / 18 \\
- \\
21 / 10 / 18\end{array}$ & $55 \%$ & $44 \%$ & $47 \%$ & $38 \%$ & $38 \%$ & $81 \%$ & $51 \%$ \\
\hline $\begin{array}{l}22 / 10 / 18 \\
- \\
28 / 10 / 18\end{array}$ & $65 \%$ & $50 \%$ & $46 \%$ & $53 \%$ & $53 \%$ & $79 \%$ & $58 \%$ \\
\hline $\begin{array}{l}29 / 10 / 18 \\
- \\
02 / 11 / 18\end{array}$ & $59 \%$ & $43 \%$ & $45 \%$ & $56 \%$ & $56 \%$ & $78 \%$ & $56 \%$ \\
\hline & & & & & & & $54 \%$ \\
\hline
\end{tabular}

Tabla 4 Indicador disponibilidad Fuente: Elaboración propia

La tabla 4 muestra la disponibilidad de las maquinas en cada semana, en promedio es de $54 \%$, lo que quiere decir, es que de 100 minutos de operación de las máquinas de la línea A y B, solo se están aprovechando $54 \mathrm{~min}$, los otros 46 minutos están siendo perdidos por tiempo muerto por mantenimiento, producción o externas. En términos más simples si el turno es de 8 horas $* 60 \mathrm{~min}=480 \mathrm{~min}-30 \mathrm{~min}$ de comida $=450 \mathrm{~min}^{*} 3$ turnos por día $=1,350 \mathrm{~min}$, sin tomar en cuenta otros factores de tiempo que pudieran intervenir en la pérdida de tiempo. Estaríamos hablando que de esos 1350 minutos que las líneas disponen para operación, se están aprovechando 729 minutos y los 621 minutos que restan son tiempo muerto.

La siguiente tabla presenta la estimación del ritmo de producción de las líneas A y B:

\begin{tabular}{|c|c|c|c|c|c|c|c|}
\hline Semana & L- 2 & G- A & G-B & S- $\mathbf{A}$ & S- B & Tamg. & Ritmo \\
\hline $\begin{array}{l}01 / 10 / 18 \\
- \\
07 / 10 / 18\end{array}$ & $83 \%$ & $91 \%$ & $35 \%$ & $59 \%$ & $59 \%$ & $94 \%$ & $70 \%$ \\
\hline $\begin{array}{l}08 / 10 / 18 \\
- \\
14 / 10 / 18\end{array}$ & $88 \%$ & $76 \%$ & $40 \%$ & $56 \%$ & $56 \%$ & $69 \%$ & $64 \%$ \\
\hline $\begin{array}{l}15 / 10 / 18 \\
- \\
21 / 10 / 18\end{array}$ & $73 \%$ & $79 \%$ & $81 \%$ & $59 \%$ & $59 \%$ & $71 \%$ & $70 \%$ \\
\hline $\begin{array}{l}22 / 10 / 18 \\
- \\
28 / 10 / 18\end{array}$ & $80 \%$ & $91 \%$ & $86 \%$ & $72 \%$ & $72 \%$ & $70 \%$ & $79 \%$ \\
\hline $\begin{array}{l}29 / 10 / 18 \\
- \\
02 / 11 / 18\end{array}$ & $75 \%$ & $73 \%$ & $77 \%$ & $76 \%$ & $76 \%$ & $69 \%$ & $74 \%$ \\
\hline & & & & & & & $72 \%$ \\
\hline
\end{tabular}

Tabla 5 Indicador ritmo de producción Fuente: Elaboración propia 
La tabla 5 muestra el ritmo de las maquinas en cada semana, pero en promedio el ritmo de las líneas A y B es de $72 \%$, lo que quiere decir que de 100 piezas que las máquinas de la línea A y B pueden procesar, se están procesando 72 piezas. Lo que tiene sentido ya que la disponibilidad de las maquinas no se está aprovechando del todo, esto repercute directamente en la producción.

A continuación se presenta el cálculo del índice de calidad:

\begin{tabular}{|c|c|c|c|c|c|c|c|}
\hline Semana & L-2 & G-A & $\begin{array}{l}\text { G- } \\
\text { B }\end{array}$ & $\begin{array}{l}\text { S- } \\
\text { A }\end{array}$ & S-B & $\begin{array}{l}\text { Tam } \\
\text { g. }\end{array}$ & Calidad \\
\hline $\begin{array}{l}01 / 10 / 1 \\
8 \\
07 / 10 / 1 \\
8\end{array}$ & $\begin{array}{l}100 \\
\%\end{array}$ & $100 \%$ & $\begin{array}{l}83 \\
\%\end{array}$ & $\begin{array}{l}90 \\
\%\end{array}$ & $\begin{array}{l}90 \\
\%\end{array}$ & $94 \%$ & $93 \%$ \\
\hline $\begin{array}{l}08 / 10 / 1 \\
8 \\
14 / 10 / 1 \\
8\end{array}$ & $91 \%$ & $93 \%$ & $\begin{array}{l}72 \\
\%\end{array}$ & $\begin{array}{l}98 \\
\%\end{array}$ & $\begin{array}{l}98 \\
\%\end{array}$ & $74 \%$ & $88 \%$ \\
\hline $\begin{array}{l}15 / 10 / 1 \\
8 \\
21 / 10 / 1 \\
8\end{array}$ & $88 \%$ & $86 \%$ & $\begin{array}{l}89 \\
\%\end{array}$ & $\begin{array}{l}83 \\
\%\end{array}$ & $\begin{array}{l}83 \\
\%\end{array}$ & $76 \%$ & $84 \%$ \\
\hline $\begin{array}{l}22 / 10 / 1 \\
8 \\
28 / 10 / 1 \\
8\end{array}$ & $98 \%$ & $100 \%$ & $\begin{array}{l}95 \\
\%\end{array}$ & $\begin{array}{l}86 \\
\%\end{array}$ & $\begin{array}{l}86 \\
\%\end{array}$ & $76 \%$ & $90 \%$ \\
\hline $\begin{array}{l}\begin{array}{l}29 / 10 / 1 \\
8 \\
02 / 11 / 1 \\
8\end{array} \\
\end{array}$ & $91 \%$ & $80 \%$ & $\begin{array}{l}84 \\
\%\end{array}$ & $\begin{array}{l}98 \\
\%\end{array}$ & $\begin{array}{l}98 \\
\%\end{array}$ & $74 \%$ & $87 \%$ \\
\hline & & & & & & & $88 \%$ \\
\hline
\end{tabular}

Tabla 6 Indicador calidad

Fuente: Elaboración propia

Analizando los datos anteriores, podemos ver que la disponibilidad afecto al indicador de ritmo, y en consecuencia este último afecta a la calidad, la tabla 6 representa el promedio de calidad con la que se procesan las piezas en las líneas A y B, de acuerdo a los datos del mes de octubre. La calidad es de $88 \%$ digamos que de 100 piezas procesadas 88 piezas son de calidad y 22 de ellas son destinadas a desecho y reproceso.

Finalmente con los tres indicadores anteriores podemos realizar el cálculo del OEE, el cual se presenta en la siguiente tabla:

\begin{tabular}{|c|c|c|c|c|c|c|c|}
\hline Semana & L-2 & G-A & G-B & S-A & S-B & Tamg. & OEE \\
\hline $\begin{array}{l}01 / 10 / 18 \\
- \\
07 / 10 / 18\end{array}$ & $56 \%$ & $40 \%$ & $10 \%$ & $22 \%$ & $22 \%$ & $87 \%$ & $40 \%$ \\
\hline $\begin{array}{l}08 / 10 / 18 \\
- \\
14 / 10 / 18\end{array}$ & $49 \%$ & $29 \%$ & $11 \%$ & $26 \%$ & $26 \%$ & $42 \%$ & $31 \%$ \\
\hline $\begin{array}{l}15 / 10 / 18 \\
- \\
21 / 10 / 18\end{array}$ & $35 \%$ & $30 \%$ & $34 \%$ & $18 \%$ & $18 \%$ & $44 \%$ & $30 \%$ \\
\hline $\begin{array}{l}22 / 10 / 18 \\
- \\
28 / 10 / 18\end{array}$ & $51 \%$ & $45 \%$ & $38 \%$ & $33 \%$ & $33 \%$ & $42 \%$ & $40 \%$ \\
\hline $\begin{array}{l}29 / 10 / 18 \\
- \\
02 / 11 / 18\end{array}$ & $40 \%$ & $25 \%$ & $29 \%$ & $41 \%$ & $41 \%$ & $40 \%$ & $36 \%$ \\
\hline & & & & & & & $35 \%$ \\
\hline
\end{tabular}

Tabla 7 Cálculo del OEE

Fuente: Elaboración propia

La siguiente gráfica muestra el comportamiento de los tres indicadores y del OEE durante el mes de octubre 2018:

Indicadores OEE de las lineas A y B, Octubre 2018.

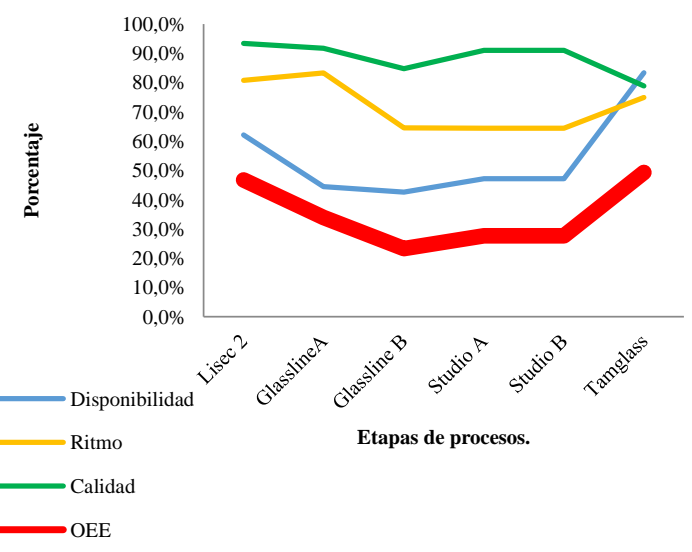

Gráfico 1 Comportamiento de indicadores y OEE Fuente: Elaboración propia

En lo general la tabla 7 nos muestra que el porcentaje de OEE del mes de octubre en las líneas de producción fue de $35 \%$, lo que se traduce en: que de 100 piezas de vidrio que las líneas podían producir, solo se produjeron 35.

En el gráfico 1, podemos observar claramente que el indicador de disponibilidad es el más bajo respecto a los demás, y esto es consecuencia de los paros de máquina y tiempos muertos, esto tendrá una repercusión directa en lo que podemos producir, en el ritmo de producción y en la calidad que se obtenga, finalmente esto se ve reflejado en un OEE bajo. 
Tomando en cuenta que la evaluación global del OEE, de acuerdo a lo mostrado en la tabla 7 , se obtuvo un rendimiento del $35 \%$, lo cual en la escala global de evaluación, representa un desempeño "inaceptable" para los procesos, lo cual representa una gran área de oportunidad para mejorar dicho desempeño.

\section{Implementación de estrategias de mejora}

Para iniciar como ya sabemos el ciclo de procesamiento de vidrio templado, de las líneas A y B inicia en el área de almacén de materia prima, posteriormente en el área de corte, esta área es la que presenta mayor tiempo de demora (ver tabla 3), en el proceso de cortar las láminas de vidrio monolítico con un tiempo de 1080 segundos. Por lo que se optó por realizar un estudio de tiempos y movimientos para analizar el proceso de corte y determinar aquellas actividades que consumen mayor tiempo por movimientos o acciones innecesarias.

A continuación se muestra el diagrama de flujo de proceso para el corte de láminas de vidrio monolítico, tomemos en cuenta que la maquina Lisec es operada por una persona y otra más se ocupa de distribuir las láminas a las líneas A y B.

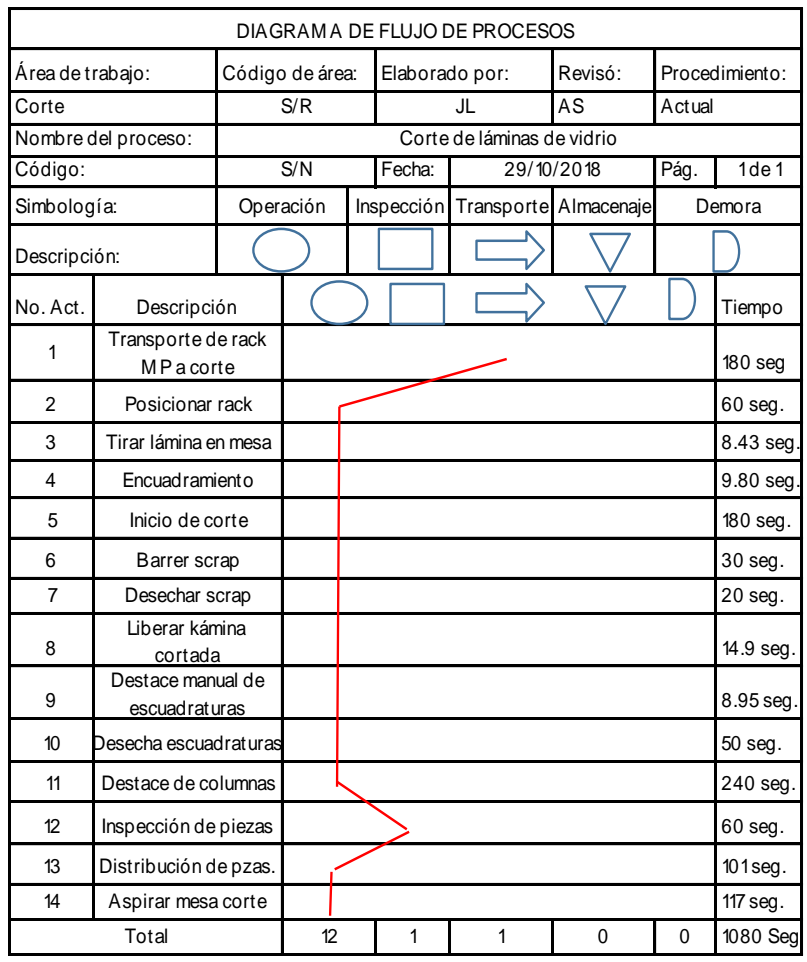

Figura 5 Diagrama de flujo de proceso, área de corte Fuente: Elaboración propia
En la figura 5, podemos observar que el paso 5 lo realiza la máquina, el operador puede aprovechar este paso para realizar el paso 6 y 7 y así ahorrarse algunos segundos. La cantidad de láminas que se pueden cortar con el tiempo ahorrado es el siguiente: los operadores tienen la capacidad de cortar 13 láminas por ciclo digamos que el ciclo dura 1080 segundos.

Entonces:

$$
\begin{aligned}
& \text { Tiempo de corte por lámina } \\
& =\frac{1080 \text { segundos }}{32 \text { láminas }} \\
& =33.75 \text { segundos por lámina. }
\end{aligned}
$$

Si se fusionan los pasos 6 y 7 con el paso 5 , nos estaríamos ahorrando 50 segundos. Esto significa que se estaría cortando 1.48 partes de una pieza de vidrio con este tiempo ahorrado.

Si se habla que durante el turno realizan un promedio de 25 ciclos:

Piezas adicionales $=25$ ciclos $*$ 1.48 piezas $=37$ piezas por turno

Con esta propuesta se estarían obteniendo 37 piezas adicionales por turno, lo cual impactará en el indicador de ritmo.

Como segunda estrategia de mejora, se identificó que la máquina G-A y G-B tiene una restricción, muy notoria ya que solo podían desfilar los vidrios, más no se podía realizar el canteado, por lo que solo se procesaban números de parte con especificaciones de acordes a esta limitante. Esta restricción retrasaba los procesos y los hacia más complejos ya que si en un día laboral había que cantear las maquinas G-A y G$B$ se quedaba paradas, perdiendo tiempo valioso para la producción.

Actualmente existen equipos G-A y G-B, que pueden realizar ambas acciones por lo que se propone la idea de adaptar un cabezal para el canteado de las piezas. Se propuso esta innovación al personal de producción, con el argumento de que podría procesar diferentes números de parte y así poder eliminar esta limitante. La respuesta fue favorable, así que el siguiente paso fue solicitar la compra y fijación del cabezal cantador al área de mantenimiento. 
Anteriormente a esta propuesta, los números de parte programados en $\mathrm{m}^{2}$ para desfilado ascendía a los $320,000 \mathrm{~m}^{2}$, con esta innovación se pretende aumentar la producción en un $40 \%$.

\section{Resultados}

Las mejoras que se realizaron en las líneas continúas A y B tuvieron efectos favorables en el sistema de indicadores. Al ahorrar tiempos, mejorar los procesos y haciendo que los espacios fueran óptimos para realizar las actividades. Cabe resaltar que las líneas deben estar en constante movimiento, con el fin de que los procesos no sean obsoletos y así poder satisfacer las especificaciones del cliente.

A continuación se muestra el indicador de disponibilidad de las líneas A y B, de las semanas 44 a 48 que comprende de las fechas 03 de noviembre al 06 de diciembre del año 2018.

\begin{tabular}{|l|l|l|l|l|l|l|l|}
\hline Semana & L-2 & \multicolumn{1}{l}{ G-A } & \multicolumn{1}{l}{ G-B } & \multicolumn{1}{l}{ S-A } & \multicolumn{1}{l|}{ S-B } & \multicolumn{1}{l|}{ Tamg. } & Disp. \\
\hline $\begin{array}{l}03 / 11 / 18 \\
-\end{array}$ & $69 / 11 / 18$ & $53 \%$ & $47 \%$ & $60 \%$ & $60 \%$ & $77 \%$ & $59 \%$ \\
\hline $\begin{array}{l}10 / 11 / 18 \\
- \\
16 / 11 / 18\end{array}$ & $80 \%$ & $55 \%$ & $48 \%$ & $90 \%$ & $90 \%$ & $79 \%$ & $74 \%$ \\
\hline $\begin{array}{l}17 / 11 / 18 \\
-23 / 11 / 18\end{array}$ & $68 \%$ & $80 \%$ & $80 \%$ & $52 \%$ & $52 \%$ & $82 \%$ & $69 \%$ \\
\hline $\begin{array}{l}24 / 11 / 18 \\
- \\
30 / 11 / 18\end{array}$ & $70 \%$ & $69 \%$ & $49 \%$ & $58 \%$ & $58 \%$ & $90 \%$ & $66 \%$ \\
\hline $\begin{array}{l}30 / 11 / 18 \\
- \\
06 / 12 / 18\end{array}$ & $73 \%$ & $57 \%$ & $50 \%$ & $53 \%$ & $53 \%$ & $82 \%$ & $61 \%$ \\
\hline
\end{tabular}

Tabla 8 Indicador disponibilidad, Noviembre Fuente: Elaboración propia.

En la tabla 8 se observa que la disponibilidad incremento en $4 \%$ más que el mes de Octubre, y eso gracias a la reducción de tiempos que se realizó en el área de corte, con 50 segundos ahorrados por turno, además de la habilitación de las bilaterales en los equipos GA y G-B, en la que ahora se aprovechan al máximo los tiempos de operación y ahora se procesan más números de parte.

Lo anterior impacta de manera directa en el indicador de ritmo producción, ya que este incrementa de manera proporcional al tiempo, por lo que se registró un aumento del $1 \%$ con respecto al mes de octubre. En la tabla 9, se presenta el comportamiento de dicho indicador:

\begin{tabular}{|c|c|c|c|c|c|c|c|}
\hline Semana & L-2 & G-A & G-B & S-A & S-B & Tamg. & Disp. \\
\hline $\begin{array}{l}03 / 11 / 18 \\
- \\
09 / 11 / 18\end{array}$ & $80 \%$ & $77 \%$ & $81 \%$ & $71 \%$ & $71 \%$ & $71 \%$ & $75 \%$ \\
\hline $\begin{array}{l}10 / 11 / 18 \\
- \\
16 / 11 / 18\end{array}$ & $82 \%$ & $82 \%$ & $77 \%$ & $70 \%$ & $70 \%$ & $74 \%$ & $76 \%$ \\
\hline $\begin{array}{l}17 / 11 / 18 \\
- \\
23 / 11 / 18\end{array}$ & $84 \%$ & $82 \%$ & $82 \%$ & $60 \%$ & $70 \%$ & $70 \%$ & $74 \%$ \\
\hline $\begin{array}{l}24 / 11 / 18 \\
- \\
30 / 11 / 18\end{array}$ & $82 \%$ & $84 \%$ & $82 \%$ & $65 \%$ & $75 \%$ & $70 \%$ & $76 \%$ \\
\hline $\begin{array}{l}30 / 11 / 18 \\
- \\
06 / 12 / 18\end{array}$ & $87 \%$ & $84 \%$ & $81 \%$ & $70 \%$ & $70 \%$ & $72 \%$ & $78 \%$ \\
\hline & & & & & & & $73 \%$ \\
\hline
\end{tabular}

Tabla 9 Indicador ritmo de producción, Noviembre Fuente: Elaboración propia

A continuación se presenta el comportamiento del indicador de calidad, el cual también presentó un incremento del $1 \%$ en relación al mes anterior, lo cual es una señal de que las estrategias implementadas son correctas.

\begin{tabular}{|l|l|l|l|l|l|l|l|}
\hline Semana & L-2 & \multicolumn{1}{l}{ G-A } & \multicolumn{1}{l}{ G-B } & \multicolumn{1}{l|}{ S-A } & \multicolumn{1}{l|}{ S-B } & \multicolumn{1}{l|}{ Tamg. } \\
\hline $\begin{array}{l}03 / 11 / 18 \\
-\end{array}$ & $90 \%$ & $85 \%$ & $90 \%$ & $88 \%$ & $88 \%$ & $75 \%$ & $86 \%$ \\
\hline $\begin{array}{l}10 / 11 / 18 \\
- \\
16 / 11 / 18\end{array}$ & $94 \%$ & $93 \%$ & $88 \%$ & $80 \%$ & $80 \%$ & $90 \%$ & $87 \%$ \\
\hline $\begin{array}{l}17 / 11 / 18 \\
- \\
23 / 11 / 18\end{array}$ & $96 \%$ & $90 \%$ & $91 \%$ & $67 \%$ & $67 \%$ & $76 \%$ & $81 \%$ \\
\hline $\begin{array}{l}24 / 11 / 18 \\
- \\
30 / 11 / 18\end{array}$ & $96 \%$ & $91 \%$ & $88 \%$ & $98 \%$ & $98 \%$ & $90 \%$ & $93 \%$ \\
\hline $\begin{array}{l}30 / 11 / 18 \\
- \\
06 / 12 / 18\end{array}$ & $100 \%$ & $100 \%$ & $96 \%$ & $98 \%$ & $98 \%$ & $77 \%$ & $95 \%$ \\
\hline
\end{tabular}

Tabla 10 Indicador calidad, Noviembre Fuente: Elaboración propia

La siguiente tabla representa el comportamiento de los tres indicadores antes mencionados, así como la determinación del OEE

\begin{tabular}{|c|c|c|c|c|c|c|c|}
\hline Indicador & L-2 & G-A & G-B & S-A & S-B & $\begin{array}{l}\text { Tam } \\
\text { g. }\end{array}$ & $\begin{array}{l}\text { Líne } \\
\text { as A } \\
\text { y B }\end{array}$ \\
\hline $\begin{array}{l}\text { Disponibili } \\
\text { dad }\end{array}$ & $\begin{array}{l}66.5 \\
\%\end{array}$ & $\begin{array}{l}53.2 \\
\%\end{array}$ & $\begin{array}{l}48.1 \\
\%\end{array}$ & $\begin{array}{l}53.7 \\
\%\end{array}$ & $\begin{array}{l}53.9 \\
\%\end{array}$ & $\begin{array}{l}84.1 \\
\%\end{array}$ & $60 \%$ \\
\hline Ritmo & $\begin{array}{l}81.5 \\
\%\end{array}$ & $\begin{array}{l}83.1 \\
\%\end{array}$ & $\begin{array}{l}69.7 \\
\%\end{array}$ & $\begin{array}{l}64.4 \\
\%\end{array}$ & $\begin{array}{l}66.3 \\
\%\end{array}$ & $\begin{array}{l}74.6 \\
\%\end{array}$ & $73 \%$ \\
\hline Calidad & $\begin{array}{l}94.7 \\
\%\end{array}$ & $\begin{array}{l}92.5 \\
\%\end{array}$ & $\begin{array}{l}86.5 \\
\%\end{array}$ & $\begin{array}{l}89.0 \\
\%\end{array}$ & $\begin{array}{l}89.0 \\
\%\end{array}$ & $\begin{array}{l}81.5 \\
\%\end{array}$ & $89 \%$ \\
\hline OEE & $\begin{array}{l}50 \\
\%\end{array}$ & $\begin{array}{l}41 \\
\%\end{array}$ & $\begin{array}{l}31 \\
\%\end{array}$ & $\begin{array}{l}31 \\
\%\end{array}$ & $\begin{array}{l}33 \\
\%\end{array}$ & $52 \%$ & $40 \%$ \\
\hline
\end{tabular}

Tabla 11 Determinación del OEE, Noviembre 
La siguiente gráfica muestra el desempeño obtenido en el mes de noviembre, entre los tres indicadores medidos y el OEE:

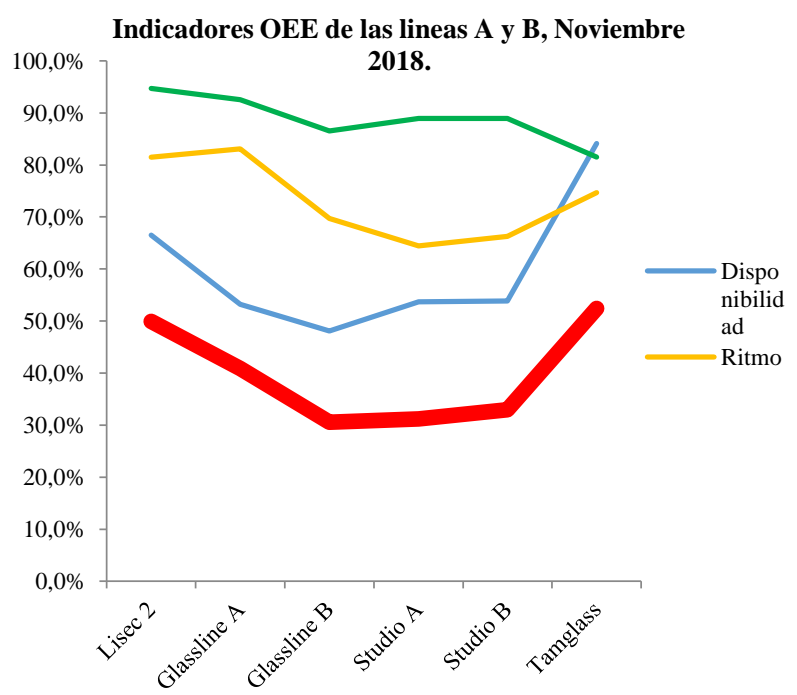

Gráfico 2 Comportamiento de indicadores y OEE Fuente: Elaboración propia.

\section{Conclusiones}

Como podemos observar se presentó un incremento del 5\% en el OEE respecto al mes anterior, esto indica que las estrategias implementadas fueron exitosas. Sin embargo la organización aún debe trabajar en acciones que le permitan mantener e incrementar el porcentaje del OEE, como podemos observar en la figura número 7, el indicador que presenta una mayor área de oportunidad es el de disponibilidad. Esto implica que se debe aprovechar al máximo el tiempo efectivo de producción de los equipos, se recomienda implementar programas de mantenimiento total productivo (TPM) que permitan reducir los tiempos de paro debidos a fallas o a intervenciones no programadas.

\section{Referencias}

Alonso G. Hugo (2009), Una herramienta de mejora, el OEE (Efectividad global de equipo), Universidad de Holguín Oscar Lucero Moya.

Belohlavek, Peter (2006), Overall Equipment Effectiveness, Blue Eagle Group, Buenos Aires

Cruelles, José (2010). La teoría de la medición del despilfarro, Torrijos, Toledo, España.

Mejía Nieto, Javier (2012). Taguchi, Revista Universidad Eafit-No. 78, Medellín.
Mora Gutiérrez, Alberto (2005), Mantenimiento estratégico para empresas industriales o de servicios, AMG, Medellín.

Ramírez Rojas, José (2009), Procedimiento para la elaboración de un análisis FODA como una herramienta de planeación estratégica en las empresas, Ciencia Administrativa 2009-2, México.

Speth, Christophe (2016), El análisis DAFO, los secretos para fortalecer su negocio, 50minutos.es, España. 